\section{Acknowledgments}

This work was supported by NIH grants AI45459, AI44374, and AI35370.

Address correspondence to: Liise-anne Pirofski, Departments of Medicine and of Microbiology and Immunology, Albert Einstein College of Medicine and Montefiore Medical Center, 1300 Morris Park Avenue, Bronx, New York 10461, USA. Phone: 718.430.2940; Fax: 718.430.8969; E-mail: 1.pirofski@einstein.yu.edu.

1. Safdar N, Bradley EA. The risk of infection after nasal colonization with Staphylococcus aureus. Am J Med. 2008;121(4):310-315.

2. Casadevall A, Pirofski L. The damage-response framework of microbial pathogenesis. Nat Rev Microbiol. 2003;1(1):17-24.

3. Fattom AI, Sarwar J, Basham L, Ennifar S, Naso R. Antigenic determinants of Staphylococcus aureus type 5 and type 8 capsular polysaccharide vaccines. Infect Immun. 1998;66(10):4588-4592.

4. Maira-Litran T, Kropec A, Goldmann D, Pier GB. Biologic properties and vaccine potential of the staphylococcal poly- $\mathrm{N}$-acetyl glucosamine surface polysaccharide. Vaccine. 2004;22(7):872-879.

5. Shinefield $\mathrm{H}$, et al. Use of a Staphylococcus aureus conjugate vaccine in patients receiving hemodialysis. N Engl J Med. 2002;346(7):491-496.

6. Creech CB, Johnson BG, Alsentzer AR, Hohenboken M, Edwards KM, Talbot TR III. Vaccination as infection control: a pilot study to determine the impact of Staphylococcus aureus vaccination on nasal carriage. Vaccine. 2009;28(1):256-260.
7. Rupp ME, et al. Phase II, randomized, multicenter, double-blind, placebo-controlled trial of a polyclonal anti-Staphylococcus aureus capsular polysaccharide immune globulin in treatment of Staphylococcus aureus bacteremia. Antimicrob Agents Chemother. 2007;51(12):4249-4254.

8. Benjamin DK, et al. A blinded, randomized, multicenter study of an intravenous Staphylococcus aureus immune globulin. J Perinatol. 2006;26(5):290-295.

9. Skurnik D, et al. Animal and human antibodies to distinct Staphylococcus aureus antigens mutually neutralize opsonic killing and protection in mice. J Clin Invest. 2010;120(9):3220-3233.

10. Robbins JB, Schneerson R, Szu SC. Perspective: hypothesis: serum IgG antibody is sufficient to confer protection against infectious diseases by inactivating the inoculum. J Infect Dis. 1995;171(6):1387-1398.

11. Maira-Litran T, Kropec A, Goldmann DA, Pier GB. Comparative opsonic and protective activities of Staphylococcus aureus conjugate vaccines containing native or deacetylated Staphylococcal Poly$\mathrm{N}$-acetyl-beta-(1-6)-glucosamine. Infect Immun. 2005;73(10):6752-6762.

12. Ohlsen K, Lorenz U. Immunotherapeutic strategies to combat staphylococcal infections [published online ahead of print May 21, 2010]. Int J Med Microbiol. doi:10.1016/j.ijmm.2010.04.015.

13. Leslie M. Biomedical research. Immunology uncaged. Science. 2010;327(5973):1573.

14. Jerne NK. The generative grammar of the immune system. EMBO J. 1985;4(4):847-852.

15. Nasu H, Chia DS, Knutson DW, Barnett EV. Naturally occurring human antibodies to the $\mathrm{F}\left(\mathrm{ab}^{\prime}\right) 2$ portion of IgG. Clin Exp Immunol. 1980;42(2):378-386.

16. Roux KH, Tankersley DL. A view of the human idiotypic repertoire. Electron microscopic and immunologic analyses of spontaneous idiotype-antiidiotype dimers in pooled human IgG. J Immunol.
1990;144(4):1387-1395

17. Shoenfeld Y. The idiotypic network in autoimmunity: antibodies that bind antibodies that bind antibodies. Nat Med. 2004;10(1):17-18.

18. Baumgarth N, Tung JW, Herzenberg LA. Inherent specificities in natural antibodies: a key to immune defense against pathogen invasion. Springer Semin Immunopathol. 2005;26(4):347-362.

19. Geha RS. Presence of circulating anti-idiotype-bearing cells after booster immunization with tetanus toxoid (TT) and inhibition of anti-TT antibody synthesis by auto-anti-idiotypic antibody. J Immunol. 1983;130(4):1634-1639.

20. Liu YD, Chen X, Enk JZ, Plant M, Dillon TM, Flynn GC. Human IgG2 antibody disulfide rearrangement in vivo. J Biol Chem. 2008;283(43):29266-29272.

21. Yoo EM, Wims LA, Chan LA, Morrison SL. Human IgG2 can form covalent dimers. J Immunol. 2003;170(6):3134-3138

22. Kelly-Quintos C, Cavacini LA, Posner MR, Goldmann D, Pier GB. Characterization of the opsonic and protective activity against Staphylococcus aureus of fully human monoclonal antibodies specific for the bacterial surface polysaccharide poly- $\mathrm{N}$-acetylglucosamine. Infect Immun. 2006;74(5):2742-2750

23. Kropec A, et al. Poly-N-acetylglucosamine production in Staphylococcus aureus is essential for virulence in murine models of systemic infection. Infect Immun. 2005;73(10):6868-6876.

24. Yoong P, Pier GB. Antibody-mediated enhancement of community-acquired methicillin-resistant Staphylococcus aureus infection. Proc Natl Acad Sci US A. 2010;107(5):2241-2246.

25. Matthias KA, Roche AM, Standish AJ, Shchepetov M, Weiser JN. Neutrophil-toxin interactions promote antigen delivery and mucosal clearance of Streptococcus pneumoniae. J Immunol. 2008;180(9):6246-6254

\title{
From skin cells to hepatocytes: advances in application of iPS cell technology
}

\author{
Linda E. Greenbaum
}

Thomas Jefferson University School of Medicine, Philadelphia, Pennsylvania, USA.

\begin{abstract}
The discovery several years ago that fibroblasts and other somatic cells from mice and humans can be reprogrammed to become inducible pluripotent stem (iPS) cells has created enthusiasm for their potential applications in regenerative medicine and for modeling human diseases. Two independent studies in this issue of the JCI provide evidence that iPS cells represent a promising source of hepatocytes for a wide range of applications, including cell transplantation, drug toxicity testing, patient-specific disease modeling, and even ex vivo gene therapy. But how far have we come?
\end{abstract}

\section{Limitations of hepatocytes for study} and treatment of human liver disease Chronic liver disease is a significant worldwide cause of morbidity and mortality in

Conflict of interest: The author has declared that no conflict of interest exists.

Citation for this article: J Clin Invest. 2010; 120(9):3102-3105. doi:10.1172/JCI44422. both pediatric and adult patient populations. Although liver transplantation has markedly reduced the burden for patients with end-stage liver disease, the shortage of organ donors and the morbidity associated with long-term immunosuppression has led to intense interest in identifying alternative therapeutic approaches, including cellular transplantation therapy.
Although hepatocyte transplantation has been achieved successfully in some patients with acute liver failure and certain metabolic diseases $(1,2)$, the use of hepatocytes for this clinical application has met several obstacles, including the need for large numbers of hepatocytes and an observed loss of differentiation during culture. Similar obstacles have been encountered with attempts to use hepatocytes for in vitro drug toxicology assays and to model human liver diseases $(3,4)$. Human embryonic and fetal stem cells can be propagated for extended periods in culture and can be differentiated to hepatocyte-like cells that are able to survive in vivo (5-8). However, the ethical issues associated with their use and their limited availability have reduced enthusiasm for this approach. 


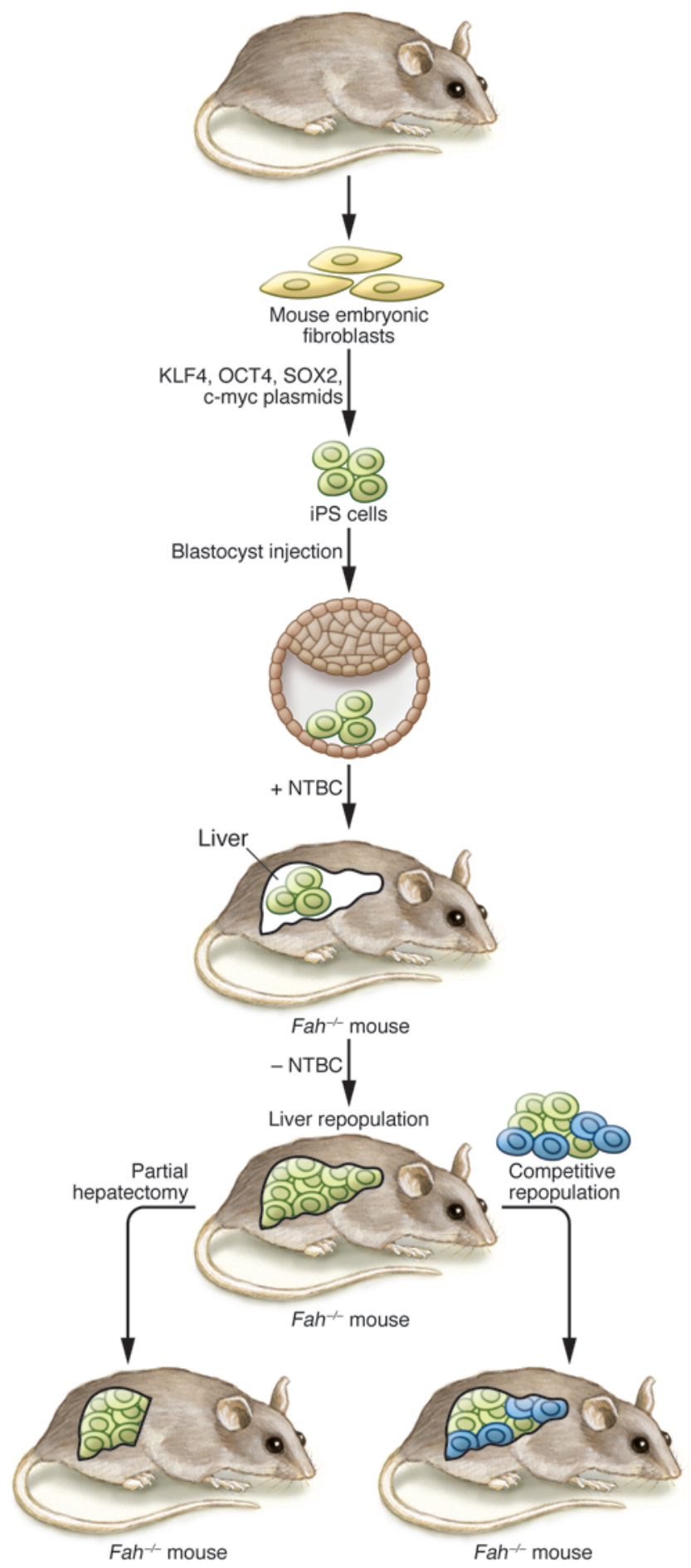

Inducible pluripotent stem cells as an alternative source of human hepatocytes

Yamanaka and colleagues first demonstrated in 2006 that introduction of 4 transcription factors, Kruppel-like factor 4 (Klf4), Octamer 3/4 (Oct4), SRY box-containing protein 2 (Sox2), and c-Myc, could effectively reprogram mouse fibroblasts to become pluripotent stem cells, which are known as inducible pluripotent stem (iPS) cells (9). This was followed a year later by the successful derivation of human iPS cells $(10,11)$. Recently, several groups reported that iPS cells can be successfully differentiated into hepatocyte-like cells (12-15) and that these cells are able to repopulate the livers of both immuno-

\section{Figure 1}

iPS cell-derived hepatocytes restore liver function in the $\mathrm{Fah}^{-/-}$mouse model of liver failure. In their study, Espejel and colleagues investigated the potential for fibroblast-derived iPS cells to proliferate and restore liver function in the $\mathrm{FAH}$-deficient liver failure model (18). Mouse embryonic fibroblasts were reprogrammed to become iPS cells using a plasmid method to deliver the reprogramming transcription factors KLF4, OCT4, SOX2, and c-myc. iPS cells were then injected into blastocysts from $\mathrm{Fah}^{-/}$mice, resulting in chimeric mice. The injected iPS cells differentiated in vivo to become hepatocytes. Upon withdrawal of NTBC in the postnatal period, $\mathrm{Fah}^{-/-}$hepatocytes died, resulting in repopulation of the liver by the transplanted iPS cell-derived (wild-type) hepatocytes and restoration of normal liver function. The authors further tested the proliferative capacity of the iPS cell-derived hepatocytes by demonstrating that these cells proliferated in response to partial hepatectomy and repopulated a $\mathrm{Fah}^{-/}$ liver as efficiently as did wild-type hepatocytes in a competitive repopulation assay.

deficient and immunocompetent mouse strains (12). Although these cells expressed many of the functions associated with fully mature hepatocytes in culture, their ability to restore liver function in models of liver disease was not tested.

Another promising application of iPS cell-derived hepatocytes is the modeling of genetic diseases in vitro using cells from individual patients. This approach could ultimately make it possible to understand the effects of specific mutations on disease pathogenesis. Hepatocytes obtained from patient iPS cells could also be used as a platform for drug hepatotoxicity assays and to individualize patient therapies. To date, few neurological disease-specific phenotypes have been modeled using patientspecific iPS cells $(16,17)$.

\section{iPS cells are highly proliferative and can restore liver function in a model of liver failure}

Two independent studies published in this issue of the JCI provide exciting data that expand our understanding of the capabilities of iPS cell-derived hepatocytes in vivo (18) and our ability to model human liver diseases using patient-specific iPS cell-derived hepatocytes (19). Espejel and colleagues tested not only whether hepatocytes differentiated from mouse iPS cells were able to repopulate the liver when transplanted, but whether these cells were sufficiently functional to restore liver function in mice 


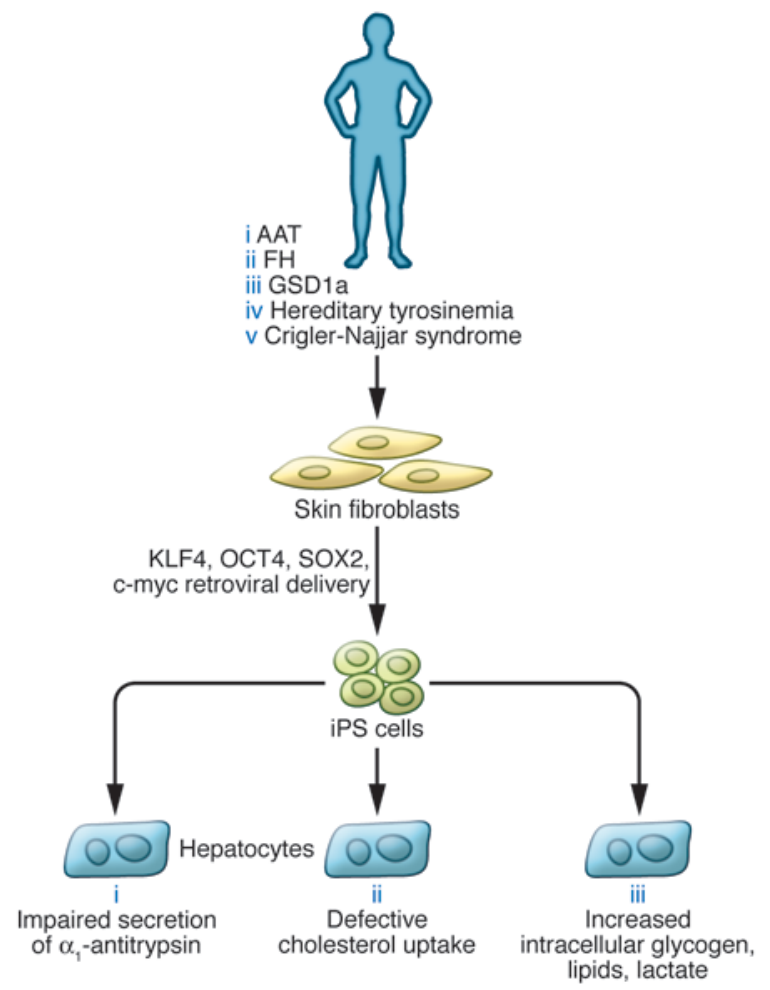

Figure 2

Genetic diseases of the liver modeled in iPS cell-derived human hepatocytes. Rashid and colleagues investigated the potential to model inborn genetic diseases of the liver in human iPS cell-derived hepatocytes (19). Skin fibroblasts were obtained from patients with 5 inborn metabolic liver diseases: AAT, FH, GSD1a, hereditary tyrosinemia, and Crigler-Najjar syndrome. Fibroblasts were reprogrammed to become iPS cells by introducing retroviral vectors expressing KLF4, OCT4, SOX2, and c-myc. iPS cells from 3 of these diseases were then differentiated in culture to become hepatocytes and analyzed for defects specific to each genetic disease.

that lack the enzyme fumarylacetoacetate hydrolase (FAH) (18), which is encoded by the gene that is mutated in human hereditary tyrosinemia. Patients that lack this essential enzyme, which is required for tyrosine metabolism, develop liver failure, neurologic impairment, and hepatocellular carcinoma as a consequence of excessive accumulation of tyrosine in these tissues. FAH-deficient mice can be maintained on the drug 2-(2-nitro-4-fluoromethylbenzoyl)-1,3-cyclohexanedione (NTBC), which blocks an enzymatic step upstream of FAH and thus prevents the accumulation of hepatotoxic metabolites. Subsequent NTBC withdrawal results in rapid liver failure. This elegant system allowed the investigators to assess how well mouse iPS cellderived hepatocytes restored liver function, since survival of the FAH-mutant animal depended upon repopulation and sufficient expression of FAH to rescue the genetic defect. The authors implanted iPS cells directly into FAH-deficient blastocysts and then waited to withdraw NTBC until the postpartum period, allowing these cells to differentiate in vivo but without providing a repopulation advantage during gestation (Figure 1). Mice with substantial chimerism, or contribution from the injected iPS cells, exhibited marked liver population by FAHexpressing hepatocytes and had liver function restored at postnatal day 22. Normally, hepatocyte function and cell number reach final size by postnatal day 21 . FAH-positive (i.e., iPS cell-derived) hepatocytes completely replaced FAH-deficient hepatocytes by postnatal day 70 , which indicated that the iPS cells continued to proliferate until full repopulation was achieved. As further proof that these cells functioned comparably to fully differentiated hepatocytes, liver function and plasma amino acid profiles continued to be normal up to postnatal day 300 (i.e., 10 months without NTBC). The iPS cell-derived hepatocytes even exhibited polyploidy, a characteristic of differentiated hepatocytes.
Espejel and colleagues also tested the long-term proliferative capacity of iPS cell-derived hepatocytes by demonstrating their capability to proliferate in response to partial hepatectomy (18). Since iPS cellderived hepatocytes possess a proliferative advantage over Fab-null hepatocytes in this context, the authors performed an even more robust test of iPS cell-derived hepatocyte proliferative capacity by transplanting equal numbers of iPS cell-derived and wild-type hepatocytes into the liver of an FAH-deficient mouse. Under these competitive conditions, the iPS cell-derived and wild-type hepatocytes contributed equally to recipient liver (Figure 1), demonstrating that iPS cell-derived hepatocytes can proliferate as well as normal hepatocytes. Because previous studies showed that fusion of hematopoietic cells with FAH-deficient hepatocytes allowed FAH-deficient hepatocytes to repopulate the liver and restore liver function (20-22), the investigators had to exclude the possibility of cell fusion as a trivial explanation for the success of the iPS cells. Using elegant genetic marker analysis, the authors showed that the overwhelming majority of hepatocytes were indeed directly derived from iPS cells, without fusion with host hepatocytes.

In summary, Espejel and colleagues extend our understanding of both the proliferative and the metabolic potential of iPS cell-derived hepatocytes in the mouse by using more robust challenges than reported previously (18). Importantly, they produced highly differentiated hepatocytes using a reprogramming technique that does not require viral integration of exogenous DNA, an important prerequisite for transplantation of human iPS cells or their derivatives into patients. An important limitation of their approach, however, is that the implantation of iPS cells directly into the early developing embryo allowed these cells to differentiate in response to developmental signals in vivo, which may have contributed substantially to their functionality. This strategy will not be feasible when applied to human iPS cells, which will need to undergo differentiation in vitro prior to transplantation. Further studies will also be needed to investigate the repopulation capabilities of iPS cell-derived hepatocytes in models in which selection advantage for the iPS cell-derived hepatocyte is weaker, more closely resembling the intrahepatic environment found in patients with chronic liver disease. 


\section{Human iPS cell-derived hepatocytes recapitulate key aspects of genetic liver disease}

In a related study also in this issue, Rashid and colleagues investigated the potential of human iPS cell-derived hepatocytes to model inborn liver diseases (19). The authors obtained skin fibroblasts from a small cohort of patients diagnosed with $\alpha_{1}$-antitrypsin deficiency (AAT), glycogen storage disease type 1a (GSD1a), familial hypercholesterolemia $(\mathrm{FH})$, hereditary tyrosinemia, or Crigler-Najjar syndrome as well as healthy controls (Figure 2). Multiple iPS cells were successfully derived from each patient using the retroviral reprogramming protocol developed by Yamanaka and colleagues (10). Next, Rashid and colleagues used a 3-step in vitro differentiation protocol using defined factors to differentiate the iPS cells sequentially into definitive endoderm, hepatic progenitor, and hepatocyte populations. The authors were able to characterize iPS cell-derived hepatocytes from patients from 3 of the 5 diseases for which iPS cells were obtained. These diseases, AAT, GSD1a, and FH, represent 3 different mechanisms of liver disease: defective protein secretion, lack of a critical intracellular enzyme, and absence of a cell surface receptor, respectively. Remarkably, the iPS cell-derived hepatocytes exhibited characteristic phenotypic abnormalities observed in native hepatocytes from patients with these 3 diseases (Figure 2). While these results are preliminary, they establish an important proof of concept and set the stage for more extensive analyses of genetic liver diseases in vitro.

The development of human iPS cellderived hepatocyte models of liver diseases will also create new opportunities to screen drugs tailored to the individual patient and to develop autologous cell transplantation therapy to correct genetic defects. The authors acknowledge some of the limitations of using iPS cell-derived patient hepatocytes to model liver diseases (19). The pathogenesis of many liver diseases involves signals from other cell populations within and outside the liver. For example, in those diseases in which delayed onset and/or environmental influences are important factors in pathogenesis, additional modifications, such as DNA damage, may be necessary in order to accurately model the disease in vitro. The authors also acknowledge that although hepatocyte function was achieved with the differentiation protocol used for this study, the persistent expression of $\alpha$-fetoprotein indicated that the iPS cell-derived hepatocytes were not fully differentiated. Additional optimization of differentiation protocols may be necessary in order to achieve full maturation of human iPS cell-derived hepatocytes. Another caveat of their study is that although the use of retroviral vectors to express reprogramming genes may be adequate for in vitro studies, alternative methods that either do not require viral transgenes for reprogramming or effectively remove these exogenous gene sequences prior to transplantation will be necessary in order to transplant iPS cell-derived hepatocytes into patients. In the course of inducing differentiation of iPS cells to hepatocytes, Rashid and colleagues observed substantial patient-topatient differences in the efficacy of the procedure. Therefore, differences in expression of the mutant protein were more likely to be caused by variability in the extent of differentiation achieved rather than by phenotypic severity of the disease. In light of these findings, caution must be used in distinguishing phenotypic differences from incomplete differentiation. Analysis of larger numbers of patients and perhaps more robust differentiation protocols will likely be necessary in order to resolve this issue.

\section{Summary}

These studies have extended our understanding of the capabilities of iPS cells for cell replacement therapy and demonstrate that key aspects of genetic liver diseases can be modeled in vitro. Substantial obstacles must be overcome, however, before iPS cells will be ready for in vivo applications. The most pressing goals include the development of efficient and consistent virus-free reprogramming methods and elimination of undifferentiated iPS cells. The potential to apply these technologies in patient-specific manners will no doubt expand the application of personalized medicine to the field of liver diseases in the near future.

\section{Acknowledgments}

The author acknowledges financial support from NIH grants R01 06669 and R01 087958.

Address correspondence to: Linda E. Greenbaum, 306 Bluemle Life Sciences Building, Thomas Jefferson University Col- lege of Medicine, 233 S. 10th Street, Philadelphia, Pennsylvania 19107, USA. Phone: 215.503.6345; Fax: 215.503.6282; E-mail: Linda.greenbaum@jefferson.edu.

1. Fitzpatrick E, Mitry RR, Dhawan A. Human hepatocyte transplantation: state of the art. J Intern Med. 2009;266(4):339-357.

2. Soto-Gutierrez A, Navarro-Alvarez N, Yagi H, Yarmush ML. Stem cells for liver repopulation. Curr Opin Organ Transplant. 2009;14(6):667-673.

3. Enns GM, Millan MT. Cell-based therapies for metabolic liver disease. Mol Genet Metab. 2008;95(1-2):3-10.

4. Fisher RA, Strom SC. Human hepatocyte transplantation: worldwide results. Transplantation. 2006; 82(4):441-449.

5. Agarwal S, Holton KL, Lanza R. Efficient differentiation of functional hepatocytes from human embryonic stem cells. Stem Cells. 2008;26(5):1117-1127.

6. Cai J, et al. Directed differentiation of human embryonic stem cells into functional hepatic cells. Hepatology. 2007;45(5):1229-1239.

7. Lavon N, Yanuka O, Benvenisty N. Differentiation and isolation of hepatic-like cells from human embryonic stem cells. Differentiation. 2004;72(5):230-238.

8. Basma $\mathrm{H}$, et al. Differentiation and transplantation of human embryonic stem cell-derived hepatocytes. Gastroenterology. 2009;136(3):990-999.

9. Takahashi K, Yamanaka S. Induction of pluripotent stem cells from mouse embryonic and adult fibroblast cultures by defined factors. Cell. 2006; 126(4):663-676.

10. Takahashi $\mathrm{K}$, et al. Induction of pluripotent stem cells from adult human fibroblasts by defined factors. Cell. 2007;131(5):861-872.

11. Yu J, et al. Induced pluripotent stem cell lines derived from human somatic cells. Science. 2007;318(5858):1917-1920.

12. Gai H, et al. Generation of murine hepatic lineage cells from induced pluripotent stem cells. Differentiation. 2010;79(3):171-181.

13. Si-Tayeb K, et al. Highly efficient generation of human hepatocyte-like cells from induced pluripotent stem cells. Hepatology. 2010;51(1):297-305.

14 . Song Z, et al. Efficient generation of hepatocytelike cells from human induced pluripotent stem cells. Cell Res. 2009;19(11):1233-1242.

15. Sullivan GJ, et al. Generation of functional human hepatic endoderm from human induced pluripotent stem cells. Hepatology. 2010;51(1):329-335.

16. Aurich $\mathrm{H}$, et al. Hepatocyte differentiation of mesenchymal stem cells from human adipose tissue in vitro promotes hepatic integration in vivo. Gut. 2009;58(4):570-581.

17. Thomson JA, et al. Embryonic stem cell lines derived from human blastocysts. Science. 1998; 282(5391):1145-1147.

18. Espejel S, et al. Induced pluripotent stem cellderived hepatocytes have the functional and proliferative capabilities needed for liver regeneration in mice. J Clin Invest. 2010;120(9):3120-3126.

19. Rashid ST, et al. Modeling inherited metabolic disorders of the liver using human induced pluripotent stem cells. J Clin Invest. 2010;120(9):3127-3136.

20. Wang $X$, et al. Cell fusion is the principal source of bone-marrow-derived hepatocytes. Nature. 2003;422(6934):897-901.

21. Willenbring $\mathrm{H}$, et al. Myelomonocytic cells are sufficient for therapeutic cell fusion in liver. Nat Med. 2004;10(7):744-748.

22. Faggioli F, Sacco MG, Susani L, Montagna C, Vezzoni P. Cell fusion is a physiological process in mouse liver. Hepatology. 2008;48(5):1655-1664. 